\title{
Miniaturization of Microwave Biosensor for Non-invasive Measurements of Materials and Biological Tissues
}

\author{
Satria Hardinata ${ }^{1}$, Frédérique Deshours ${ }^{1}$, Georges Alquié ${ }^{1}$, Hamid Kokabi ${ }^{1}$, Fabien Koskas ${ }^{1}$
}

\begin{abstract}
Non-invasive planar complementary split ring resonators (CSRRs) coupled to microstrip line for measuring the dielectric properties of materials and biological tissues are presented in this paper. The expectations of health professionals are increasingly turning to less invasive surgical procedures and treatments. In particular, the monitoring of vital parameters (sweat, water in the lungs, etc.) or the evolution of certain pathologies, such as cancer cells, could be observed regularly if suitable devices were developed and could especially replace traditional invasive method. Appropriate miniaturized RF or microwave devices could be an alternative for some medical diagnostic applications. These devices would make it possible to determine the dielectric characteristics of biological tissues, which represent their real pathological states. It would thus be possible, by means of dielectric contrast measurements, to follow the evolution of pathology as well as the vital parameters of a patient. The objective of this research is to produce a prototype biosensor that is suitable for measurements on biological tissues and that can be miniaturized to enhance its spatial sensitivity. This work focuses on the design, electromagnetic simulations and characterization of a new miniaturized biosensors operating between 1 and $10 \mathrm{GHz}$. The ex-vivo experimental results will be shown by measuring the S-parameters of various materials and animal biological tissues. The extraction of the dielectric parameters of these samples is obtained by the measurements of materials.
\end{abstract}

Keywords-Microwave Biosensor, Non-Invasive Measurement, Complex Permittivity, Ring Resonator, SParameters, Resonant Frequency.

\section{INTRODUCTION}

Knowledge of the dielectric properties of biological tissues at microwave frequencies is very important for several reasons. It is linked to the development of wireless communications. Indeed, the fact that man is increasingly exposed to multiple electromagnetic sources in outdoor (LTE, GPS, ...) and indoor (WiFi, Bluetooth, ...) can be a source of physiological discomforts and even diseases. It is therefore important to know the interactions between the electromagnetic field and the human body. Another reason is the development of wireless embedded microwave sensors on the human body to perform "human

${ }^{1}$ Satria Hardinata, Frédérique Deshours, Georges Alquié, Hamid Kokabi, and Fabien Koskas are with Sorbonne Universités, UPMC Univ Paris 06, UR2, L2E, F-75005, Paris, France. E-mail: satria.hardinata@etu.upmc.fr; frederique.deshours@upmc.fr; georges.alquié@upmc.fr; hamid.kokabi@upmc.fr; fabien.kokas@upmc.fr. monitoring", i.e. to measure vital human parameters such as post-operative follow-up or physical activity.

Complex permittivity is one of the most important parameters of biological tissues in RF and microwave engineering. The response of the microwave planar circuit depends strongly on the dielectric properties of the material used as the substrate. The measurements of complex permittivity have also been explored in the field of health.

Several microwave characterization techniques have been developed for the measurement of the permittivity of Materials Under Test (MUT). These methods can be classified into three categories: free space characterization technique, transmission line technique (waveguides, coaxial cable) for which the insertion of the MUT modifies the propagation and the so-called resonant methods based on a resonator coupled by transmission lines. Among these characterization techniques, the most accurate method is the resonant method The variation of the resonance frequency and the quality factor of the resonator containing the material under test gives information on the dielectric properties of the material. Recently, devices coupling a planar ring resonator to microstrip lines have been proposed to determine the dielectric constant of various materials. The planar technology makes it possible to design two types of ring resonators, either in distributed elements or in lumped elements which allow to obtain structures of reduced size.

The design of microwave planar sensors provides many advantages for medical instrumentation such as portability, ability to perform non-invasive, in-depth and non-ionizing measurements. These resonators were originally used for the characterization of low-loss materials for microelectrics. The applications for medical agro-food of these resonators are much more recent. The objective of this research is to produce a miniaturized microwave sensor prototype that is suitable for the dielectric characterization of biological tissues between 1 and $10 \mathrm{GHz}$.

The principle concept of the resonators is firstly presented in chapter II, then followed by the design of the two sensors developed on a low cost substrate (substrate FR4) in chapter III. Then, the simulation and optimization process over HFSS software is shown in chapter IV. The measurements of these unloaded and loaded sensors will be presented in chapter $\mathrm{V}$ as well as the extraction of the dielectric parameters of the materials tested. We conclude and give a perspective for this future research in terms of applications in vascular surgery. 


\section{CONCEPT OF CSRR}

Compared to usual $\lambda / 2$ transmission line resonators, the CSRR has typically dimension which is smaller than its wavelength $(\lambda / 10)$, leading to a high level of miniaturization. The CSRR is actually derived from the metamaterial basic structure Split Ring Resonator (SRR). This structure consists in two very closed concentric metal split ring resonators on a substrate. As described in Figure 1 , in the complementary split ring resonator, metals are replaced by apertures etched in the ground planem so dielectric substrate fulfilled the grooves. Material characterization method used to extract its dielectric properties is resonant method. This method observes the frequency shift and the change of quality factor due to MUT over the resonator.

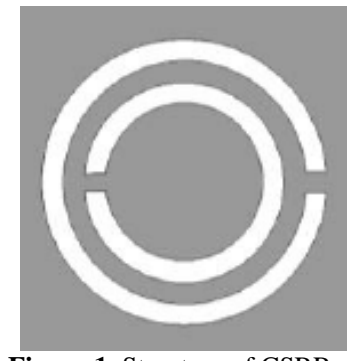

Figure 1. Structure of CSRR

\section{SENSOR DESIGN}

The configuration and the design parameters of circular and square CSRRs are shown in figure 2(a) and 2(b), respectively. The equivalent circuit of both the CSRRs could be represented by the topology shown in figure 2(c). The symbols Lc and Cc represent the inductance and capacitance of CSRR. For the equivalent circuit model shown in Figure 2(c), the resonant frequency may be given as

$$
f_{r}=\frac{1}{2 \pi \sqrt{L r+C C}}
$$

The CSRR sensors have been implemented on the FR4 substrate $\left(\varepsilon_{\mathrm{r}}=4.3, \tan \delta=0.02\right)$. The CSRR design parameters taken in the simulation are explained in table 1.

At first, both the sensors are optimized in the HFSS software, and the simulation is carried out to obtain the resonance frequency at $2.4 \mathrm{GHz}$ (ISM frequency band). It is interesting to note from Table I that the circumference value of circular CSRR is smaller than square one.

TABEL 1.

PARAMETERS FOR BOTH CSRR.

\begin{tabular}{lllll}
\hline \hline CSRR model & $\mathbf{a}(\mathbf{m m})$ & $\mathbf{g}(\mathbf{m m})$ & $\mathbf{s}(\mathbf{m m})$ & $\mathbf{t}(\mathbf{m m})$ \\
\hline Square & 7.49 & 0.4 & 0.4 & 0.4 \\
Circular & 4.54 & 0.5 & 0.5 & 0.5 \\
\hline \hline
\end{tabular}

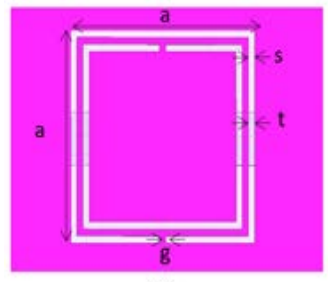

(a)

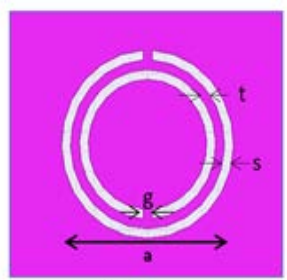

(b)

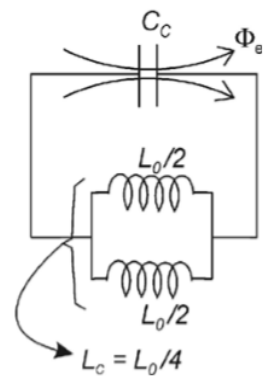

(c)

Figure 2. Design of (a) square and (b) circular CSRRs and (c) equivalent circuit model. Purple area represents conductor part and white area represents etched out part.

This means that as per equation (1), the resonant frequency of the circular CSRR will be higher than the square one. This can also be justified by the fact that the resonant frequency of such resonators actually decrease by increasing value of circumference of the CSRRs.

\section{Simulation OF SENSOR}

The simulated resonant frequency of the proposed sensor in the unloaded condition is found to be $2.4 \mathrm{GHz}$ for both models, and the quality factor is 39.4 and 88.9 respectively, for square and circular model (figure 3)

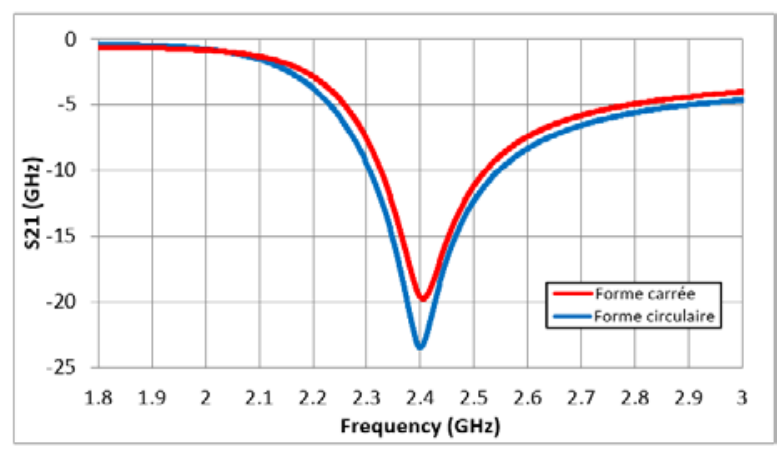

Figure 3. Simulation result of optimized CSRR at $2.4 \mathrm{GHz}$

The MUT is placed on the resonator and cover the entire area. After loading the sensor with the material under test (MUT), a change in the resonant frequency as well as in the magnitude of S21 (dB) is observed as shown in Figure 4. This is achieved by varying the dielectric properties of the MUT. During the numerical simulation, various dielectric materials with the dielectric constant ranging from $\varepsilon_{\mathrm{rs}}=1$ to 60 and $\tan \delta_{\mathrm{s}}$ varying from 0 to 0.4 are simulated to observe frequency shift and band broadening. The values of resonant frequencies and quality factors extracted from the recorded transmission coefficients, are then used to derive the numerical model with the help of a curve fitting technique. 


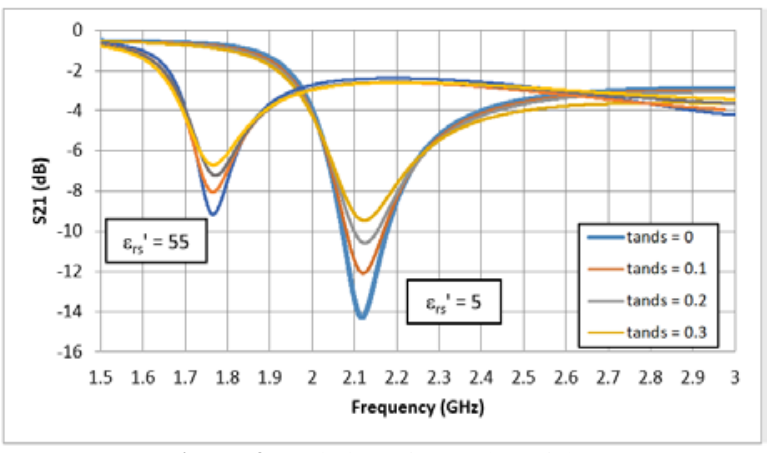

Figure 4. Variation of S21 $(\mathrm{dB})$ of the sensor

The curve fitting procedure generates the equations for the complex permittivity determination as the function of the resonant frequency and the thickness of MUT. For the square CSRR, we have

$$
\varepsilon_{\mathrm{rs}}^{\prime}=\mathrm{e}^{\frac{\mathrm{f}_{\mathrm{r}}^{-2}-0.183}{0.0024 \times \mathrm{d}+0.0207}}
$$

and for the circular model, we have

$$
\varepsilon_{r s}^{\prime}=e^{\frac{f_{r}^{-2}-0.174}{0.0041 \times d+0.0195}}
$$

\section{MEASUREMENT AND Result}

The planar sensors are fabricated with a standard photolithography technique on commercially available $0.8 \mathrm{~mm}$ FR4 substrate having a conductive copper film coating of $35 \mu \mathrm{m}$. A pair of $50 \mathrm{ohms} \mathrm{SMA} \mathrm{connectors} \mathrm{is}$ then mounted on the fabricated sensor through a mechanical welding enabling the measurement of Sparameters with a vector network analyzer (VNA). The overall sensor length is $40 \mathrm{~mm}$ and its width is $20 \mathrm{~mm}$. The measurement setup is shown in figure 5(a). The top and bottom views of the sensor are shown in figure 5(b) and 5(b), respectively.

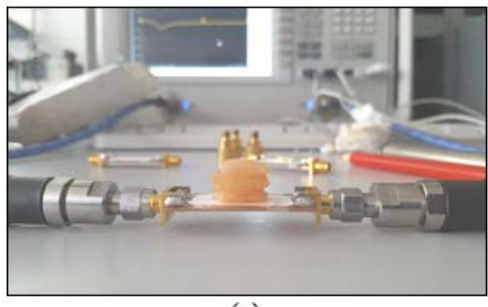

(a)

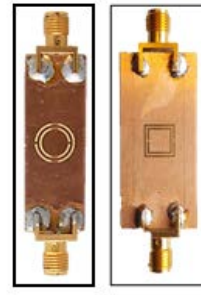

(b)

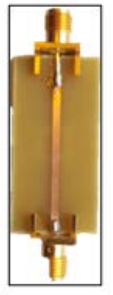

(c)
Figure 5. (a) Measurement setup, (b) Bottom view and (c) Top view of CSRR sensor

We have chosen four materials under test that represent low and high value of dielectric properties, such as FR4, DUROID, unsalted butter and chicken meat. Table II below shows the detail of their dielectric properties.

TABLE 2.

DIELECTRIC PROPERTIES OF MATERIAL UNDER TEST FROM LITERATURE

\begin{tabular}{cccc}
\hline \hline Material & $\boldsymbol{\varepsilon}_{\text {rs' }}$ & $\boldsymbol{\varepsilon}_{\text {rs }}{ }^{\prime}$ & $\tan \boldsymbol{\delta}_{\mathbf{s}}$ \\
\hline FR4 & 4.3 & 0.086 & 0.02 \\
RT DUROID & 10.2 & 0.023 & 0.0023 \\
Unsalted butter & 24.5 & 4.3 & 0.175 \\
Chicken meat & 55 & 17.3 & 0.3 \\
\hline \hline
\end{tabular}

By putting the MUT on the resonator, we have succeed to measure the material with low dielectric properties such as FR4 and RT Duroid 6010. Since we increase the value of dielectric constant and loss of MUT, we couldn't observe any frequency shift and changement of quality factor.

These measurements disagree with the simulated results because chicken meat that contains a lot of blood is in direct contact with the resonators. In the simulations, the MUT was considered a solid and not a soft body. Retrosimulations must therefore confirm the experimental results.

In order to reduce the effect induced by materials with very high constant and dielectric losses on the sensor response, we added a layer of Teflon over the resonators. By protecting the CSSR with such a thin and water-resistant thin tape, we can reduce the disturbance produced by the MUT.

The added Teflon layer has a dielectric constant of 2.1, a dissipation factor of 0.001 and a thickness of $20 \mu \mathrm{m}$. It is held with scotch tape as shown in figure 6.

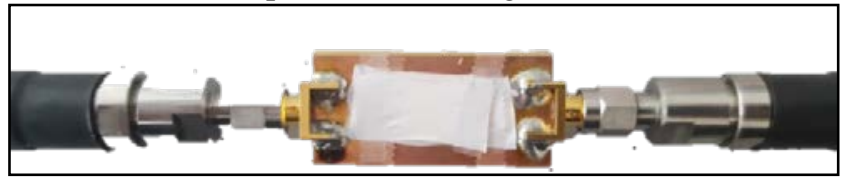

Figure 6. Protection of CSRR by a layer of Teflon

The addition of this layer has the effect of producing a shift of the resonance frequency of $15 \mathrm{MHz}$ for the squareshaped CSRR and $23 \mathrm{MHz}$ for the circular-shaped CSRR.

We have succeed then observing the frequency shift and changement of quality factor by the different thickness of chicken meat starting from 2 to $6 \mathrm{~mm}$.

We can observe the presence of resonance peaks which can be exploited for the extraction of the dielectric properties of the MUT. The proposed solution consists the adding a protective layer of Teflon thus makes it possible to solve the problem of disable resonance caused by the blood on the meat. The results obtained are summarized in Table III. It shows that the higher the thickness of the MUT, the greater the frequency shift, and so the smaller the quality factor $\left(\mathrm{Q}_{\text {мuт }}\right)$.

TABLE 3.

MEASUREMENT RESULT OF THE SENSOR LOADED BY CHICKEN MEAT

\begin{tabular}{llll}
\hline \hline Structure of CSRR & $\begin{array}{l}\text { Thickness } \\
(\mathbf{m m})\end{array}$ & $\begin{array}{l}\text { Frequency shift } \\
\text { (MHz) }\end{array}$ & $\mathbf{Q}_{\text {MUT }}$ \\
\hline \multirow{3}{*}{ Square } & 2 & 600 & 18.6 \\
& 4 & 641 & 14.3 \\
& 6 & 653 & 11 \\
\hline \multirow{3}{*}{ Circulaire } & 2 & 650 & 19.4 \\
& 4 & 700 & 10.85 \\
& 6 & 730 & 10.6 \\
\hline \hline
\end{tabular}

By the optimization under HFSS, we were able to extract the values of the dielectric constant and the dielectric losses of the chicken. The typical values of the chicken at 2.45 GHz are: $\varepsilon_{\mathrm{rs}}=55$ and $\tan \delta_{\mathrm{s}}=0.3$. Figure 7 below simply confirmed that the optimization and measurement result are match for the square model. 
The $3^{\text {rd }}$ International Seminar on Science and Technology

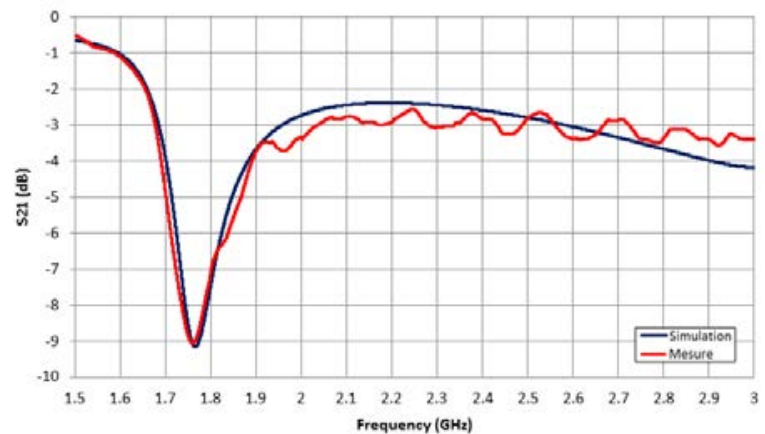

Figure 7. Simulation and measurement result graph of CSRR sensor loaded by $2 \mathrm{~mm}$ of chicken meat

\section{CONCLUSION}

During this research, complementary split ring resonators (CSRR) sensors in two models (square and circular) were fabricated in FR4 microstrip technology for the microwave characterization of dielectric materials. These resonators were optimized at $2.4 \mathrm{GHz}$ which is the frequency of the ISM band. We were confronted with an important problem during the experimentation of samples having characteristics close to those of the biological tissues. Indeed, for such materials, the resonant method using CSRRs no longer makes it possible to exploit the resonance peaks and thus to extract the dielectric parameters of the material. This problem was solved by reducing the disturbance produced by the MUT by the addition of a thin layer of Teflon protection at the resonator. The CSRR sensor could penetrate up to $6 \mathrm{~mm}$ depth of chicken meat.

The studies of the CSRR could be extended to hexagonal form to facilitate the creation of sensor network applied to medical domain. This sensor network could be applied to the skin to provide information about the vascular state and real time at a given depth. It provides also vascular mapping and imaging, not only to diagnosis but also therapy, for example as a catheterization aid.

\section{ACKNOWLEDGEMENT}

The authors would like to thank L2E Laboratory at UPMC that support us during the research. We would like to thank also LPDP Indonesia who support us financially to produce the CSRR sensor prototype on the substrate ROGERS DUROID 4003.

\section{REFERENCES}

Use the "Insert Citation" button to add citations to this document. 